\title{
1-Methylbenzvalen - Synthese und einige Reaktionen
}

\section{Manfred Christl *, Petra Kemmer und Brigitte Mattauch ${ }^{1)}$}

Institut fur Organische Chemie der Universitat Wurzburg, Am Hubland, D-8700 Würzburg

Eingegangen am 26. Juli 1985

Aus der Reaktion von Cyclopentadienyllithium mit 1,1-Dichlorethan und $n$-Butyllithium ging in praparativ brauchbaren Mengen 1-Methylbenzvalen (1) hervor. Seine Hydrierung mit Diimin erbrachte 1-Methyltricyclo[3.1.0.02,6]hexan (3). Benzonitriloxid und 2,4,6-Trimethylbenzonitriloxid addierten sich an die Doppelbindung von 1 unter Bildung von Gemischen der isomeren $\Delta^{2}$-Isoxazolin-Derivate 4 und 5. 4-Methyl-4H-1,2,4-triazol-3,5-dion ergab mit 1 die umgelagerten Addukte 6-8. Schon bei $20^{\circ} \mathrm{C}$ wandelte sich 8 langsam in das DihydrodiazasemibullvalenDerivat 9 um. Durch Einsatz von [6-D]-1-Methylbenzvalen (1a) wurden die Mechanismen der zu 6 -9 fuhrenden Reaktionen untersucht.

\section{1-Methylbenzvalene - Synthesis and Several Reactions}

1-Methylbenzvalene (1) has been obtained in preparatively useful quantities from the reaction of cyclopentadienyllithium with 1,1-dichloroethane and $n$-butyllithium. Hydrogenation of 1 by means of diimine afforded 1-methyltricyclo[3.1.0.0 $0^{2,6}$ ]hexane (3). Addition of benzonitrile oxide and 2,4,6-trimethylbenzonitrile oxide to the double bond of 1 gave rise to the formation of a mixture of the isomeric $\Delta^{2}$-isoxazoline derivatives 4 and 5. 4-Methyl- $4 H-1,2,4-$ triazole-3,5-dione and 1 reacted to give the rearranged adducts $6-8$. At temperatures as low as $20^{\circ} \mathrm{C} 8$ transformed slowly into the dihydrodiazasemibullvalene derivative 9. Using [6-D]-1-methylbenzvalene (1a) the mechanisms of the reactions leading to $6-9$ have been studied.

Aufgrund der guten Zugănglichkeit durch die Synthese von Katz et al.2) konnte die Chemie des Benzvalens ${ }^{3)}$ intensiv untersucht werden. Dabei entdeckte man neuartige Reaktionen ${ }^{3-5)}$, und die Nutzung des Synthesepotentials gestattete die Darstellung lange gesuchter Molekule 3,6). Zwar sind einige substituierte Benzvalene bekannt 3,5,7-9), jedoch erschweren die Lange der Synthesewege oder maßige Ausbeuten das Studium der Eigenschaften. Burger et al. ${ }^{8)}$ entwickelten zwei Routen zum 1-Methylbenzvalen (1). Die Umsetzung von 5-(Chlormethyl)-5-methyl-1,3-cyclopentadien mit Schlosser-Base in Gegenwart von [18]-Krone-[6] lieferte neben 1 eine kleine MengeSpiro[4.2]hepta-2,4-dien sowie Toluol. Nachteilig ist die Stufenzahl bei bescheidenen Ausbeuten bei den zwei letzten Schritten. Der andere Weg zu 1 besteht in der Methylierung von 1-Lithiobenzvalen, wobei ein Gemisch aus 1 und Benzvalen resultiert.

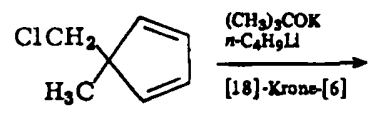

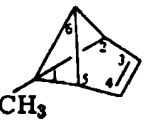

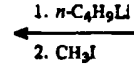

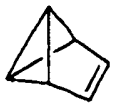

(C) VCH Verlagsgesellschaft mbH, D-6940 Weinheim, 1986 0009-2940/86/0303-0960\$02.50/0 


\section{A. 1-Methylbenzvalen (1) und sein Dihydroderivat 3}

Die Strategie von Katz et al. ${ }^{2)}$ zur Synthese von Benzvalen laßt sich in zwei Varianten auf die Darstellung von Methylbenzvalenen übertragen. Burger et al. ${ }^{8)}$ gingen von Methylcyclopentadienyllithium aus und erzielten durch Umsetzung mit Dichlormethan und Methyllithium ein Gemisch aus 2- und 3-Methylbenzvalen sowie Toluol. Wir ließen jetzt 1,1-Dichlorethan und Butyllithium in Ether oder Hexan auf Cyclopentadienyllithium einwirken und kamen zu einer Lösung, in der mit Ausbeuten von 13-28\% 1, $3-5 \%$ Toluol und $38-44 \%$ 2-Hexen vorlagen.
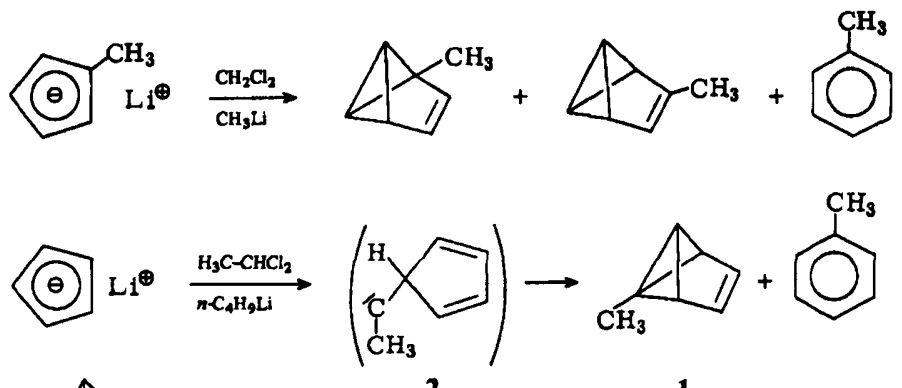<smiles>CC1C2CC13C1CC2CC13</smiles>

In Analogie zum Mechanismus der Bildung von Benzvalen ${ }^{8)}$ ist anzunehmen, daß das durch Metallierung von 1,1-Dichlorethan ${ }^{10)}$ entstehende 1,1-Dichlor-1-lithioethan mit Cyclopentadienyllithium reagiert und nach Abspaltung von 2 Molekülen Lithiumchlorid Cyclopentadienylmethylcarben (2) hervorbringt, in dem die 1,4-Addition ${ }^{8}$ ) des Carbenzentrums an das Diensystem - möglich, aber weniger wahrscheinlich, ist auch die 1,2-Addition - zu 1 führt. Toluol resultiert aus der Umlagerung von 2 unter [1,2]-C-Wanderung. H-Wanderungsprodukte wie 6-Methylfulven oder Vinylcyclopentadiene fanden wir nicht. Zur Bildung von 2-Hexen, das durch seine Reaktionsprodukte 17 und 18 mit 4-Methyl-4H-1,2,4-triazol-3,5-dion identifiziert wurde, nehmen wir an, daß Butyllithium mit Cyclopentadienyllithium um 1,1-Dichlor-1-lithioethan konkurriert ${ }^{11)}$ und $n$-Butylmethylcarben generiert, das der H-Verschiebung unterliegt.

Nach der für Benzvalen ausgearbeiteten Vorschrift ${ }^{12)}$ haben wir 1 mit Diimin hydriert und durch präparative Gaschromatographie 15\% 1-Methyltricyclo[3.1.0.02,6]hexan (3) isoliert.

Wie im unsubstituierten Benzvalen ${ }^{3,13)}$ absorbieren $6-\mathrm{H}(\delta=3.71)$ sowie $\mathrm{C}-1(59.2)$ und C-6 (53.6) von 1 bei ungewohnlich niedrigem Feld, was der Vergleich mit 3 (6-H: $\delta=1.81 ; \mathrm{C}-1$ : 11.5; C-6: 7.4) besonders klar vor Augen führ. Zur Deutung dieses Phänomens haben wir ein Orbitalmodell vorgeschlagen ${ }^{13,14)}$. In Tab. 1 sind die ${ }^{13} \mathrm{C}$-NMR-Parameter von 1 und 3 sowie die der zugehörigen unsubstituierten Kohlenwasserstoffe zusammengestellt. Aus den chemischen Verschiebungen lassen sich Substituenteneffekte einer Methylgruppe an einer Bicyclo[1.1.0]butan-Brückenkopfposition ableiten: $\alpha=10.9,9.1, \beta=5.3,5.0$ (C-6) und 3.2, 3.1 (C-2,5), $\gamma=-0.9,0.2 \mathrm{ppm}$. Sie stimmen mit den aus Tricyclo[4.1.0.0.7. derivat erhaltenen Werten sehr gut überein ${ }^{15)}$.

Chem. Ber. 119 (1986) 
Tab. $1 .{ }^{13} \mathrm{C}$-NMR-Chemische Verschiebungen ( $\delta$-Werte) und ${ }^{13} \mathrm{C}$-H-Kopplungskonstanten uber eine Bindung (in $\mathrm{Hz}$, jeweils zweite Zeile) von Benzvalen, 1-Methylbenzvalen (1) und ihren Dihydroderivaten in $\mathrm{CDCl}_{3}$ mit TMS als internem Standard

\begin{tabular}{lccccc}
\hline Verbindung & $\mathrm{C}-1$ & $\mathrm{C}-2,5$ & $\mathrm{C}-3,4$ & $\mathrm{C}-6$ & $\mathrm{CH}_{3}$ \\
\hline Benzvalen $^{\text {a) }}$ & 48.3 & 36.6 & 133.7 & 48.3 & - \\
& 205.2 & 168.8 & 172.8 & 205.2 & \\
1 & 59.2 & 39.8 & 132.6 & 53.6 & 11.9 \\
& $-\mathrm{b})$ & $167 \mathrm{~b})$ & $\left.172^{\mathrm{c}}\right)$ & $204 \mathrm{~d})$ & $128 \mathrm{c})$ \\
Tricyclo- & 2.4 & 34.0 & 26.1 & 2.4 & - \\
{$\left[3.1 .0 .0^{2.6}\right]-$} & 212 & 163 & 132 & 212 & \\
hexan? & 11.5 & 37.1 & 26.3 & 7.4 & 10.3 \\
3 & - & 165 & 136 & 215 & 127 \\
\hline
\end{tabular}

a) Lit. 13), - b) Feinstruktur m. - c) Feinstruktur q, $6 \mathrm{~Hz}$ - d) Keine Feinstruktur. - e) Feinstruktur d, $6 \mathrm{~Hz}$. - ${ }^{-1}$ Lit. ${ }^{12)}$, Lösungsmittel $\mathrm{C}_{6} \mathrm{D}_{6}$.

\section{B. Reaktionen von 1 mit Nitriloxiden und 4-Methyl-4H-1,2,4-triazol-3,5-dion}

Nitriloxide addieren sich mit hohen Ausbeuten an Benzvalen"). Bei 1 stellte sich die Frage nach der Stereochemie: Würde die der Methylgruppe zu- oder abgewandte Seite der Doppelbindung bevorzugt angegriffen?
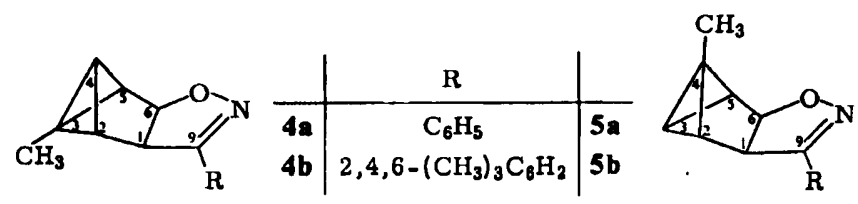

Die Gegenwart von erheblichen Mengen 2-Hexen störte die Reaktion von 1 mit Benzo- und 2,4,6-Trimethylbenzonitriloxid nicht, d.h. in der Konkurrenz ist 1, wie erwartet ${ }^{9,16)}$, weit uberlegen. Es bildeten sich mit sehr guten Ausbeuten die Isomeren 4 und 5 in Verhalltnissen nahe 1:1. Damit übt die Methylgruppe so gut wie keinen dirigierenden Effekt aus. Da sie wegen der Geometrie des Bicyclo[1.1.0]butan-Systems die Doppelbindung kaum sterisch abschirmt, dürfte auch der elektronische Einfluß auf beiden Seiten der Doppelbindung ăhnlich, d.h. klein sein.

Im Falle der Phenylverbindungen 4a und 5a gelang die spezifische Zuordnung der beiden Signalsătze in den ${ }^{1}$ H-NMR-Spektren nicht. Dagegen ließen die Feinstrukturen der Banden bei den Mesitylderivaten $\mathbf{4 b}$ und $\mathbf{5 b}$, die durch Kristallisation in reiner Form bzw. stark angereichert erhalten wurden, die Einzelcharakterisierungen zu. Dabei wurde wie in früheren Beispielen $6 d$ ) vorausgesetzt, daß 1- und 6-H aufgrund der günstigeren Bindungsanordnung mit 4-H eine groBere Fernkopplung aufweisen sollten als mit 3-H. Wir weisen daher die Doppeltripletts bei $\delta=3.82$ und $5.15 \mathrm{mit} J_{1,6}=8.4$ und $J_{1,2}=J_{1,4}=1.2$ bzw. $J_{4,6}=J_{5,6}=1.0 \mathrm{~Hz} 1-\mathrm{H} \mathrm{bzw} .6-\mathrm{H}$ von $4 \mathrm{~b}$ zu. Die entsprechenden Absorptionen von $5 \mathrm{~b}$ enthalten neben $J_{1,6}$ höchstens eine Kopplungskonstante der Größenordnung $1 \mathrm{~Hz}$. Dieses Kriterium war in den Spektren von 4a, 5a nicht anwendbar, weil die sehr ähnlichen chemischen Verschiebungen von 2- und 5-H die Feinstrukturen komplizieren.

Chem. Ber. 119 (1986) 
Aus der Reaktion von Benzvalen mit 4-Phenyl-4H-1,2,4-triazol-3,5-dion ${ }^{17}$ bzw. der 4-Methylverbindung (MTAD) ${ }^{18)}$ gehen ganz einheitlich die 7,8-Diazatetracyclo[3.3.0.0 $0^{2,4} \cdot 0^{3.6}$ ]octan-Derivate hervor, die zur Synthese von Prisman ${ }^{17)}$ und Dewarbenzol ${ }^{18)}$ genutzt worden sind. 1-Methylbenzvalen verhält sich anders. Wir identifizierten als Produkte seiner Umsetzung mit MTAD die Urazole 6, 7 und 8 im Verhältnis 1.0:2.6:1.0 mit einer Gesamtausbeute von 37\%.

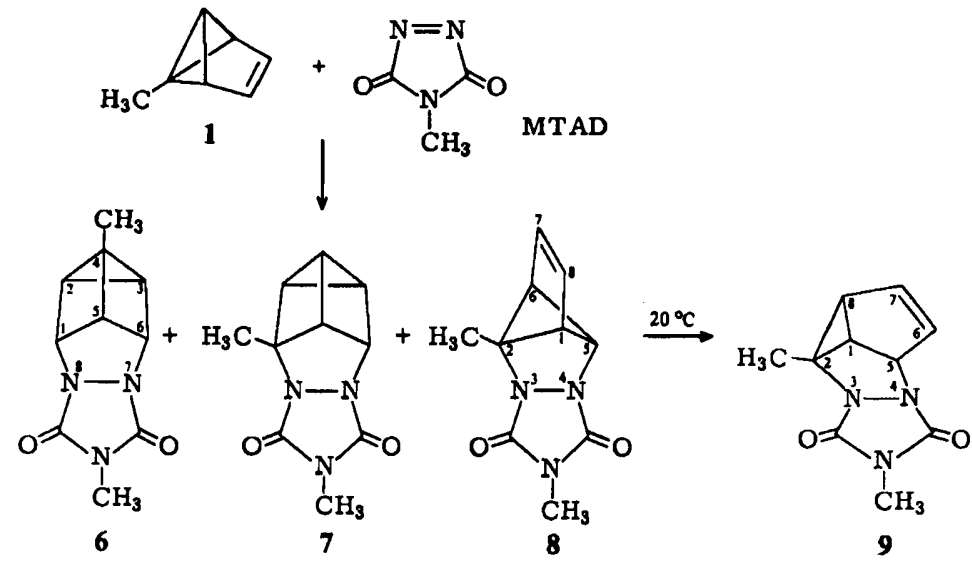

Das vierte Isomere 9 entstand durch Umlagerung von 8 bei $20^{\circ} \mathrm{C}$ mit einer Halbwertszeit von mehreren Tagen in Losung bzw. mehreren Monaten im festen Zustand. Da wir Verbindungen mit derartigen Kohlenstoff-Gerusten zuvor ausführlich untersucht hatten ${ }^{4 d, 19)}$, fiel die Charakterisierung von 6-9 nicht schwer. Besondere Beachtung verdienen die chemischen Verschiebungen von $5-\mathrm{H}(\delta=5.25), \mathrm{C}-2(\delta=105.98)$ und $C-5(\delta=91.05)$ in 8 . Diese ungewðhnlichen Tieffeldwerte gehen auf die Natur der betreffenden C-Atome als Glieder eines starren, gefalteten Cyclopentenrings ${ }^{13)}$ zurück.

Um den Mechanismus dieser Umlagerungen aufzuhellen, vereinigten wir MTAD mit [6-D]-1-Methylbenzvalen (1a), das wir aus 1 durch Metallierung mit Butyllithium und dann Reaktion mit $\mathrm{D}_{2} \mathrm{O}$ bereiteten. Dabei gelangte die Markierung in 6 zu jeweils $50 \%$ in die Positionen 1 und 2 (siehe 6a,b), in 7 eindeutig in Position 4 (siehe 7a) und in 8 eindeutig in Position 7 (siehe 8a). Diese Ergebnisse zeigen die gleichen Wege an, die bei der Addition von Benzvalen einerseits an 4-Phenyl-4H-1,2,4-triazol-3,5-dion ${ }^{4 d, 17}$ und andererseits an Chlorsulfonylisocyanat ${ }^{4 d, 7)}$ und Tetracyanethen ${ }^{4 d)}$ genutzt werden. Die Annăherung von MTAD muß auf beide Seiten der Doppelbindung von 1 zielen. Erfolgt sie auf jene trans zur Methylgruppe, dann resultiert nur 6; dagegen gehen aus dem cis-Angriff 7 und 8 hervor.

Ausgehend von 1 a sollte im trans-Fall, wie fur die Benzvalen-Reaktion vorgeschlagen $^{4 d)}$, das Aziridinium-Zwitterion 10 entstehen, in dem die Wagner-MeerweinUmlagerung nur auf der dem Elektrophil-Teil abgewandten Seite eintreten kann, weil die CN-Bindung von der Rückseite her geoffnet werden muß. Im neuen Zwitterion 11 verbindet sich eines der beiden gleichwertigen kationischen Zentren der Zweikohlenstoffbrücke in der 2-Bicyclo[2.1.1]hexen-6-yl-Einheit mit dem anionischen N-Atom, und so bildet sich, da der sekundäre Isotopeneffekt vernachlässigbar ist, ein 1:1-Gemisch von 6-Molekülen mit der Marke in der 1- oder 2-Position (6a,b). Das

Chem. Ber. 119 (1986) 
Produkt der Wagner-Meerwein-Umlagerung cis-seitig zum Elektrophil-Teil in 10 besaßße das Gerüst von 8a, jedoch wăren $\mathrm{CH}_{3}$ und $\mathrm{D}$ vertauscht.

Dagegen kann ein Aziridinium-Zwitterion das Auftreten von 7 und 8 nicht erklären, weshalb die Annaherung von MTAD an 1 a cis-seitig zur Methylgruppe das Zwitterion 12 hervorbringen dürfte, das Zwischenstufen analog ist, die für Teilreaktionen von Benzvalen mit Chlorsulfonylisocyanat ${ }^{4 d, 7)}$ und Tetracyanethen ${ }^{4 d)}$ postuliert worden sind. Die [1,2]-C-Wanderung kann nun, da ein freies Carbeniumion-Zentrum vorliegt, auf der dem Elektrophil-Teil abgewandten (Weg a) oder der zugewandten Seite (Weg b) ablaufen, woraus die nichtklassischen $Z$ witterionen 13 bzw. 14 hervorgehen. Wurde die entsprechende Aziridinium-Zwischenstufe an die Stelle von 12 treten, konnte kein 14, sondern nur 13 entstehen.

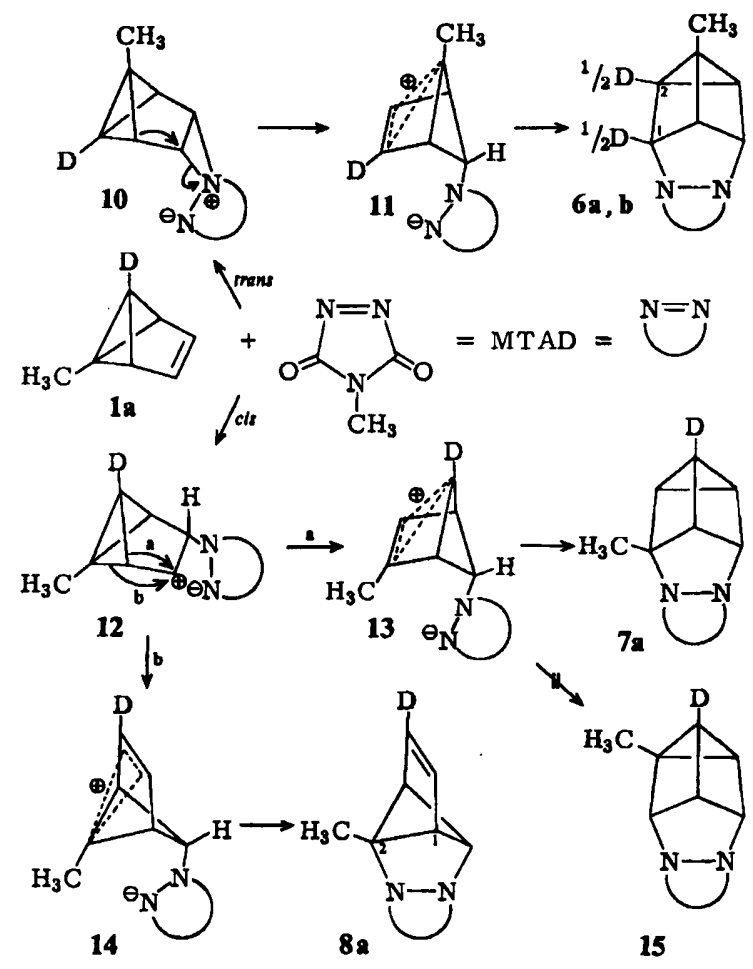

Als Ursache für die Bildung von 12 anstelle der Aziridinium-Zwischenstufe oder aus dieser sehen wir die cis-orientierte Methylgruppe, die wegen ihres Raumbedarfs den Heterocyclus nicht in der Symmetrieebene des Teilchens duldet. Aber im Falle der trans-Anordnung der Methylgruppe ist der symmetrische Zustand 10 offenbar günstig. Zwecks Knüpfung einer $\sigma$-Bindung kann in 14 das nucleophile Zentrum das elektrophile aus răumlichen Gründen nur an der Einkohlenstoffbrücke der 2-Bicyclo[2.1.1]hexen-6-yl-Einheit erreichen, so daß eindeutig 8a entsteht. Warum sich allerdings 13 ausschließlich zu 7a stabilisiert und nicht auch $\mathrm{zu} \mathrm{15}$, bleibt ebenso Gegenstand von Spekulationen wie der Befund, daß die isolierten Produkte hauptsächlich aus der Anla-

Chem. Ber. 119 (1986) 
gerung von MTAD an die zur Methylgruppe cis-orientierte Seite der 1-Doppelbindung resultieren $(\mathbf{7}+\mathbf{8})$.

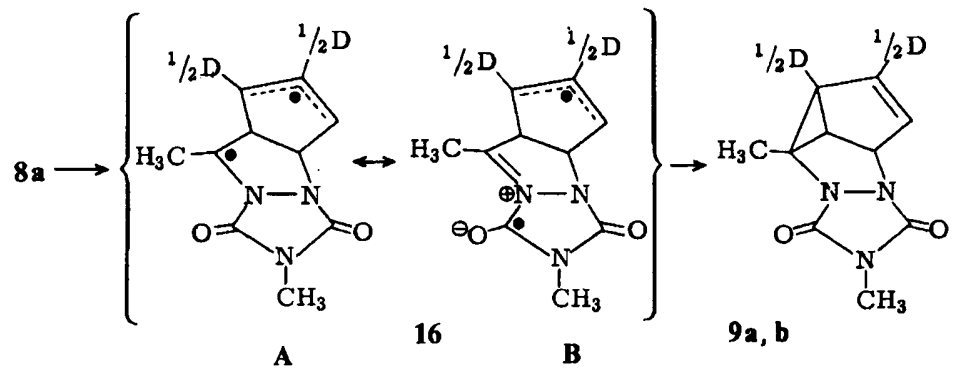

Der Übergang von 8 in 9 schon bei $20^{\circ} \mathrm{C}$ erstaunte uns, da Tricyclo[3.3.0.0.6.6 3-en erst bei ca. $150^{\circ} \mathrm{C}$ Dihydrosemibullvalen liefert ${ }^{20}$ ) und sich auch ein Tetracarbonitril-Derivat bei $20^{\circ} \mathrm{C}$ als stabil erwiesen hatte ${ }^{4 d)}$. Jedoch lagert sich Tricyclo[3.3.0.0 $0^{2,6}$ ]octa-3,7-dien bei $20^{\circ} \mathrm{C}$ rasch in Semibullvalen um ${ }^{21}$. Für diese Reaktion sind sowohl ein zweistufiger Weg über ein Diradikal ${ }^{20,21)}$ postuliert als auch ein konzertierter $\left[{ }_{\sigma}{ }_{s}+{ }_{\sigma} 2_{a}\right]$-Proze ${ }^{22}{ }^{2}$ vorgeschlagen worden. Zwischen diesen Möglichkeiten, die auch auf $8 \rightarrow 9$ angewandt werden können, zu unterscheiden, gestattete der Einsatz von 8 a. Es entstand 9a,b, d.h. die Markierung von 8 a tauchte gleichmäßig über die Positionen 7 und 8 von 9 verteilt auf. Dies ist nur mit einer suprafacialen [1,3]-C-Verschiebung in 8 (8a) unter Retention der Konfiguration am wandernden Zentrum (C-2) vereinbar, entspricht also einem gemăß den Woodward-Hoffmann-Regeln verbotenen Prozeß. Damit tritt als Zwischenstufe wahrscheinlich das Diradikal 16 auf, dessen Generierung offenbar nur einer geringen Aktivierung bedarf, weil auch der Radikalzustand im heterocyclischen Teil eine allylartige Stabilisierung genießt, wie die Mesomerieformel 16B deutlich macht. Eine zwitterionische Zwischenstufe scheidet aus, weil sich die Umlagerung in $\mathrm{CDCl}_{3}$ und $\left(\mathrm{CD}_{3}\right)_{2} \mathrm{SO}$, also in zwei Lösungsmitteln recht unterschiedlicher Polarität, mit praktisch der gleichen Geschwindigkeit vollzog. Der hochselektive Bruch der C-1-C-2- oder C-2-C-6-Bindung in 8, wobei die C-1 - C-5- und C-5 - C-6-Bindungen völlig intakt bleiben, geht vermutlich auf den sterischen Druck zurück, den die 2-Methylgruppe durch die Vinylenbrücke erfährt.

Dihydrosemibullvalen-Abkömmlinge sind auch als Produkte konzertierter 1,4-Cycloadditionen von Tetracyanethen und 5,6-Dichlor-p-benzochinon-2,3-dicarbonitril an Benzvalen gefunden worden ${ }^{4 d)}$. Die Addition von $\mathrm{SO}_{2}$ liefert auf einem zweistufigen Weg über ein $\mathrm{Zwitterion}$ ein Oxa-thia-Derivat und in einer Einstufenreaktion ein strukturell verwandtes Vierringsulfon nebeneinander ${ }^{4 d)}$. Die Bildung von 9 über 8 eröfnet die dritte Route für die Umwandlung des Benzvalen- in das Dihydrosemibullvalen-System.

Die Hexamethyl- $N$-phenyl-Verbindung mit dem Ringgerust von 8 ist aus Hexamethylprisman sowie aus Hexamethyldewarbenzol und 4-Phenyl-4H-1,2,4-triazol-3,5-dion erhalten worden, im letzteren Falle durch die Umlagerung des zu 6, 7 analogen Primäraddukts. Uber eine Weiterisomerisierung vergleichbar mit dem Übergang $8 \rightarrow 9$ wurde jedoch nichts berichtet ${ }^{23}$ ).

Die Reaktion von 1 mit MTAD wurde durch das vorhandene 2-Hexen nicht gestört, obwohl dieses mit überschüssigem MTAD die bekannten Produkte 17 und 1824) der En-Reaktion lieferte. Bei Anwendung einer zu 1 ăquivalenten MTAD-Menge konnte 2-Hexen unverăndert abdestilliert

Chem. Ber. 119 (1986) 
und dann mit MTAD umgesetzt werden. In der Konkurrenz um MTAD kommt also 2-Hexen gegen 1 nicht zum Zuge. Da NMR-Daten von 17 und 18 nicht publiziert worden sind 24), fügen wir diese im Versuchsteil an.<smiles></smiles><smiles>CCCCC1=CC=C(CCC)C(=O)NN1CCCC</smiles>

Wir danken der Deutschen Forschungsgemeinschaft und dem Fonds der Chemischen Industrie für die finanzielle Förderung und der Union Rheinische Braunkohlen Kraftstoff AG, Wesseling, fur Dimethylether.

\section{Experimenteller Teil}

\section{Gerăte9).}

1-Methyltricyclo[3.1.0.0 2,6]hex-3-en (1-Methylbenzvalen) (1): Der gesamte Versuch wurde unter sorgfältigem Ausschluß von Luft und Feuchtigkeit (Stickstoffatmosphäre, trockene Reagenzien und Lơsungsmittel) ausgeführt. Von $320 \mathrm{mmol}$ etherischem bromidfreiem Methyllithium ${ }^{25}$ ) wurde der Ether bei Normaldruck abdestilliert $(30 \mathrm{~min})$. Man kuhlte dann auf $-78^{\circ} \mathrm{C}$ und kondensierte $350 \mathrm{ml}$ Dimethylether auf das Methyllithium (1 h), erwarmte auf -35 bis $-30^{\circ} \mathrm{C}$ und tropfte $26 \mathrm{ml}$ ( $315 \mathrm{mmol}$ ) Cyclopentadien so zu, daß die Methanentwicklung nicht zu sturmisch verlief (30 min). Nach vollstandigem Abklingen der Methanentwicklung tropfte man zunächst vorsichtig, dann rasch (einige $\mathrm{min}$ ) $39.5 \mathrm{ml}$ (468 mmol) 1,1-Dichlorethan zu, wobei so gut wie kein Temperatureffekt beobachtet wurde. Das anschließende Zutropfen von $355 \mathrm{mmol}$ Butyllithium in Ether $(1.2-1.5 \mathrm{~N})$ erfolgte wegen der stark exothermen Reaktion langsam $(1-1.5 \mathrm{~h})$, und die Temp. wurde durch starkes Kühlen auf -35 bis $-30^{\circ} \mathrm{C}$ gehalten. Danach entfernte man das Kuhlbad und ließ auf Raumtemp. kommen (1.5 h). Der dabei verdampfende Dimethylether wurde zum Auffangen von mitgerissenem Produkt durch zwei hintereinandergeschaltete, auf $-20^{\circ} \mathrm{C}$ gehaltene Kolben geleitet. Diese kuhlte man nach Abdampfen des Dimethylethers auf $-78^{\circ} \mathrm{C}$ und destillierte im Wasserstrahlvakuum die fluchtigen Produkte aus dem Reaktionskolben $(1 \mathrm{~h})$. Man entfernte den ersten Kolben mit der Hauptmenge, hielt den zweiten Kolben weiterhin auf $-78^{\circ} \mathrm{C}$ und vervollstăndigte im 0.1-Torr-Vak. durch Erwärmen auf $40^{\circ} \mathrm{C}$ die Destillation. Man erhielt nach Vereinigung der Fraktionen 300-350 ml Destillat, das hauptsächlich aus Ether und Dimethylether bestand. Die weiteren Komponenten wurden mit Ausnahme des ubriggebliebenen 1,1-Dichlorethans mit Mesitylen als internem Standard anhand der Integrale ihrer ${ }^{1} \mathrm{H}$-NMR-Signale quantitativ bestimmt (es sind die Extremausbeuten bei mehreren Versuchen angegeben): $3.8-8.2 \mathrm{~g} 1(13-28 \%), 0.8-1.5 \mathrm{~g}$ Toluol $(3-5 \%)$, und 11.6-13.2 g 2-Hexen (38-44\%, bezogen auf Butyllithium). Diese Lossungen wurden bei den unten beschriebenen Versuchen direkt verwendet. Zur Messung der NMR-Spektren von 1 engten wir $30 \mathrm{ml}$ der Losung bei $0^{\circ} \mathrm{C}$ im 60-Torr-Vak, bis auf ca. $3 \mathrm{ml}$ ein (nicht weiter, da 1 in reinem Zustand detonieren dürfte), fugten $5 \mathrm{ml} \mathrm{CDCl}{ }_{3}$ zu, engten wieder zum Teil ein und wiederholten diese Prozedur. Es lag dann eine $\mathrm{CDCl}_{3}$-Losung ahnlicher Mengen an 1, Toluol und Ether vor.

${ }^{1} \mathrm{H}-\mathrm{NMR}\left(\mathrm{CDCl}_{3}\right)$ von $1: \delta=1.71\left(\mathrm{~s} ; \mathrm{CH}_{3}\right), 1.94(\mathrm{q}$, Linienabstand $1.5 \mathrm{~Hz} ; 2-, 5-\mathrm{H}), 3.71(\mathrm{t}$, $\left.J_{2.6}=1.5 \mathrm{~Hz} ; 6-\mathrm{H}\right), 5.85(\mathrm{t} ; 3-, 4-\mathrm{H})$. Burger et al.8) gaben Werte an, die wegen eines anderen Losungsmittels (Ether $/ C_{6} D_{6} 9: 1$ ) um ca. $0.1 \mathrm{ppm}$ nach hoherem Feld verăndert sind. ${ }^{13}$ C-NMR: Tab. 1. 
Mit ähnlichem Erfolg setzten wir anstelle von Butyllithium in Ether auch Butyllithium in Hexan ein, wobei eine Hexanlosung von 1, Toluol, 2-Hexen und 1,1-Dichlorethan anfiel.

[6-D]-1-Methyltricyclo/3.1.0.0.0.6 Jhex-3-en (1 a): Bei $0^{\circ} \mathrm{C}$ und unter Stickstoff gab man $1.90 \mathrm{~g}$ $(20.6 \mathrm{mmol}) 1$ in $30 \mathrm{ml}$ Ether zu $175 \mathrm{ml} 1 \mathrm{~N} n$-Butyllithium in Ether und ließ $15 \mathrm{~h}$ bei $0^{\circ} \mathrm{C}$ ruhren. Durch vorsichtige Zugabe von $8 \mathrm{ml} \mathrm{D}_{2} \mathrm{O}$ zersetzte man die Lithium-organischen Verbindungen vollständig, filtrierte unter Kühlung des Filtrats auf $-15^{\circ} \mathrm{C}$ einen Feststoff ab und wusch mit wenig kaltem Ether nach. Nach Trocknen uber $\mathrm{K}_{2} \mathrm{CO}_{3} / \mathrm{Na}_{2} \mathrm{SO}_{4}$ bei $0^{\circ} \mathrm{C}$ gab man $1.5 \mathrm{~g}$ Natriumhydrid zu und rührte $2 \mathrm{~h}$ bei $-15^{\circ} \mathrm{C}$. Dann kondensierte man die fluchtigen Anteile i. Vak., zuletzt bei 0.1 Torr, in eine auf $-78^{\circ} \mathrm{C}$ gekuhlte Vorlage und ermittelte $608 \mathrm{mg}(32 \%) 1 \mathrm{a}$ in $200 \mathrm{ml}$ Ether. Den Deuteriumgehalt bestimmte man anhand der Massenspektren von 9 und $9 \mathbf{a}, \mathbf{b}$ zu $98.5 \%$.

1-Methyltricyclo/3.1.0.02.6 Jhexan (3): $2.6 \mathrm{~g}$ (28.2 mmol) 1 in $170 \mathrm{ml}$ Ether, der von der 1-Synthese her noch 1,1-Dichlorethan, Toluol und 2-Hexen enthielt, wurden mit $25 \mathrm{~g}$ Hydrazinhydrat, $24 \mathrm{~g}$ 2-Aminoethanol und $100 \mathrm{ml}$ Ethylenglycolmonomethylether gemischt und unter starkem Rühren innerhalb von $2 \mathrm{~h}$ tropfenweise mit $150 \mathrm{~g} \mathrm{~K}_{3} \mathrm{Fe}(\mathrm{CN})_{6}$ in $300 \mathrm{ml}$ heißem Wasser versetzt, wobei man durch Kuhlung mit Eis eine Innentemp. von $6-9^{\circ} \mathrm{C}$ aufrechterhielt. Das Ende der Reaktion erkannte man am Verschwinden des faulen 1-Geruchs und durch das Fehlen der Bande von 1 bei $\delta=5.85 \mathrm{im}{ }^{1} \mathrm{H}$-NMR-Spektrum der Etherphase, die die wäßrige Suspension von $\mathrm{K}_{4} \mathrm{Fe}(\mathrm{CN})_{6}$ überschichtete. Im Wasserstrahlvak. kondensierte man bis $2 \mathrm{u} 80^{\circ} \mathrm{C}$ Badtemp. die flüchtigen Komponenten einschließlich eines Teils Wasser in eine auf $-78^{\circ} \mathrm{C}$ gekühlte Vorlage um. Man trennte die Phasen des Kondensats, extrahierte die Wasserphase $2 \mathrm{mal} \mathrm{mit} \mathrm{je} 20 \mathrm{ml}$ Ether, trocknete die vereinigten Etherphasen ubber $\mathrm{K}_{2} \mathrm{CO}_{3}$, versetzte sie mit $2 \mathrm{~g} \mathrm{NaH}$ (Gasentwicklung) und destillierte die Hauptmenge des Ethers uber eine Drehbandkolonne ab. Aus dem Ruckstand wurden die fluchtigen Anteile im Wasserstrahl- und dann im Olpumpenvak. in gekuhlte Vorlagen umkondensiert, zur Entfernung von Resten protonenaktiver Substanzen mit $\mathrm{LiAlH}_{4}$ versetzt und erneut umkondensiert. Mittels präparativer Gaschromatographie (6-m-Săule, 5\% $\mathrm{NaOH}$ und 20\% Carbowax auf Chromosorb P/NAW) trennte man restlichen Ether, Toluol, 1,1-Dichlorethan und 2-Hexen ab, so daß $400 \mathrm{mg}(15 \%)$ reines 3 als farblose Flüssigkeit anfielen. - IR (Gasphase): 3040 (s), 2950 (s), 2880 (s), 1468 (w), 1460 (w), 1450 (w), 980 (m), 893 (w), $793(\mathrm{~m}), 691 \mathrm{~cm}^{-1}(\mathrm{w}) .-\mathrm{MS}(70 \mathrm{eV}): m / z=94\left(37 \%, \mathrm{M}^{+}\right), 93(14), 91(22), 79$ (100), 77 (46), 65 (9), 51 (10). - ' $\mathrm{H}-\mathrm{NMR}\left(\mathrm{CDCl}_{3}\right): \delta=1.58$ (br. s; 3-, 4- $\mathrm{H}_{2}$ ), 1.81 (br. s; 6-H), $1.87\left(\mathrm{~s} ; \mathrm{CH}_{3}\right)$, 2.15 (br. s; 2-, 5-H). - ${ }^{13}$ C-NMR: Tab. 1.

$$
\mathrm{C}_{7} \mathrm{H}_{10} \text { (94.2) Ber. C } 89.30 \mathrm{H} 10.70 \text { Gef. C } 89.31 \text { H } 10.92
$$

3-Methyl- (4a) und 4-Methyl-9-phenyl-7-oxa-8-azatetracyclo/4.3.0.0.4.4. $0^{3.5}$ Jnon-8-en (5a): $\mathrm{Zu}$ $550 \mathrm{mg}(6.0 \mathrm{mmol}) 1$ und $930 \mathrm{mg}(9.18 \mathrm{mmol})$ Triethylamin in $10 \mathrm{ml}$ Hexan tropfte man unter Kühlung mit Eis und unter Rühren innerhalb von $30 \mathrm{~min}$ die Losung von $710 \mathrm{mg}$ (4.57 mmol) Benzhydroximoylchlorid 26) in $3 \mathrm{ml}$ Ether und ruhrte weitere $6 \mathrm{~h}$ im Eisbad. Dann fugte man soviel Wasser zu, daß sich der farblose Niederschlag aufloste, trennte die Phasen, extrahierte die wăßrige Phase mit $10 \mathrm{ml}$ Ether, trocknete die vereinigten organischen Phasen uber $\mathrm{Na}_{2} \mathrm{SO}_{4} /$ $\mathrm{K}_{2} \mathrm{CO}_{3}$ und engte i. Vak., zuletzt bei 0.01 Torr, ein. Der gelbe Ruckstand wog $1.0 \mathrm{~g}$ und bestand aus einem nur wenig verunreinigten, teilkristallinen $57: 43$-Gemisch aus $4 \mathrm{a}$ und $5 \mathrm{a}$ (Ausb. quantitativ). Bei der Behandlung mit $n$-Hexan gingen die Kristalle in Lơsung und ein Öl blieb ungelöst. Man dekantierte die Hexan-Lösung, engte sie i. Vak. ein, nahm den Rückstand in wenig Chloroform auf, setzte der Losung etwas $n$-Hexan zu und bewahrte bei $-20^{\circ} \mathrm{C}$ auf, wobei sich farblose Kristalle mit Schmp. $79-83^{\circ} \mathrm{C}$ abschieden, die sich als Gemisch mit ca. $90 \%$ Überschußisomerem erwiesen. - ${ }^{1} \mathrm{H}$-NMR $\left(\mathrm{CDCl}_{3}\right)$ : Überschußisomeres: $\delta=1.46\left(\mathrm{~s} ; \mathrm{CH}_{3}\right), 2.06$ (br. s; 3-H oder 4-H), 2.30 (br. s; 2-, 5-H), 3.77 (br. d, $J_{1,6}=8.9 \mathrm{~Hz} ; 1-\mathrm{H}$ ), 5.04 (br. d; 6-H), $7.2-7.5$ (m; 3 aromat. H), 7.6-7.9 (m; 2 aromat. H). Unterschußisomeres: $\delta=1.50\left(\mathrm{~s} ; \mathrm{CH}_{3}\right), 1.90$ (br. s;

Chem. Ber. 119 (1986) 
4-H oder 3-H), 2.30 (br. s; 2-, 5-H), 3.86 (br. d, $J_{1,6}=8.9$ Hz; 1-H), 5.04 (br. d; 6-H), $7.2-7.5$ ( $\mathrm{m} ; 3$ aromat. $\mathrm{H}), 7.6-7.9(\mathrm{~m} ; 2$ aromat. $\mathrm{H})$.

$$
\mathrm{C}_{14} \mathrm{H}_{13} \mathrm{NO}(211.3) \text { Ber. C } 79.59 \text { H } 6.20 \text { N } 6.63 \text { Gef. C } 80.14 \text { H } 6.35 \text { N } 6.73
$$

3-Methyl- (4b) und 4-Methyl-9-(2,4,6-trimethylphenyl)-7-oxa-8-azatetracyclol4.3.0.0.0,4.03,5)non-8-en (5 b): $2.00 \mathrm{~g}$ (12.4 mmol) 2,4,6-Trimethylbenzonitriloxid 27 ) wurden bei $0^{\circ} \mathrm{C}$ in $25 \mathrm{ml}$ Hexan, das $1.40 \mathrm{~g}(15.2 \mathrm{mmol}) 1$ enthielt, suspendiert und $20 \mathrm{~h} \mathrm{im} \mathrm{Eisbad} \mathrm{gerührt.} \mathrm{Man} \mathrm{filtrierte}$ dann einen geringfügigen Niederschlag ab und engte die Lösung i.Vak. ein. Das ${ }^{1}$ H-NMRSpektrum identifizjerte den gelben Ruckstand als 1:1-Gemisch der beiden Isomeren $4 \mathrm{~b}$ und $\mathbf{5 b}$. Die Losung des Ruckstandes in $n$-Hexan schied beim Aufbewahren bei $-30^{\circ} \mathrm{C} 340 \mathrm{mg}(11 \%) 4 \mathrm{~b}$ als farblose Kristalle mit Schmp. $118-122^{\circ} \mathrm{C}$ ab. Einengen der Mutterlauge, Aufnehmen in $n$-Hexan und Aufbewahren bei $-30^{\circ} \mathrm{C}$ erbrachte $220 \mathrm{mg}(7 \%)$ eines $4: 1$-Gemisches aus $5 \mathrm{~b}$ und 4 b mit Schmp. $87-91^{\circ} \mathrm{C}$. Der Mutterlaugenrïckstand wog $1.8 \mathrm{~g}(62 \%)$ und bestand aus praktisch reinem $1: 1-\mathrm{Gemisch}$ aus $4 \mathrm{~b}$ und $5 \mathrm{~b}$. $-{ }^{1} \mathrm{H}-\mathrm{NMR}\left(\mathrm{CDCl}_{3}\right): 4 \mathrm{~b}: \delta=1.57\left(\mathrm{~s} ; 3-\mathrm{CH}_{3}\right), 2.06$ und 2.43 (jeweils dm, $J_{2,5}=5 \mathrm{~Hz} ; 2-\mathrm{H}, 5-\mathrm{H}$ ), 2.08 (br. s; 4-H), 2.27 (s; 3 aromat. $\mathrm{CH}_{3}$ ), 3.82 (dt, $\left.J_{1,6}=8.4, J_{1,2}=J_{1,4}=1.2 \mathrm{~Hz} ; 1-\mathrm{H}\right), 5.15\left(\mathrm{dt}, J_{4,6}=J_{5,6}=1.0 \mathrm{~Hz} ; 6-\mathrm{H}\right), 6.90$ (br. s; 2 aromat. H). 5 b: $\delta=1.68\left(\mathrm{~s} ; 4-\mathrm{CH}_{3}\right.$ ), 2.03 (br. s; 3-H), 2.12 und 2.47 (jeweils dt, $J_{2,5}=4.8, J_{1,2}=J_{2,3}$ $\approx J_{3,5} \approx J_{5,6} \approx 1.5 \mathrm{~Hz} ; 2-\mathrm{H}$ und $\left.5-\mathrm{H}\right), 3.72$ (br. d, $J_{1,6}=8.4 \mathrm{~Hz} ; 1-\mathrm{H}$ ), 5.15 (br. d; 6-H), 6.92 (br. s; 2 aromat. H).

$$
\begin{aligned}
& \mathrm{C}_{17} \mathrm{H}_{19} \mathrm{NO} \text { (253.3) Ber. C } 80.59 \text { H } 7.56 \text { N } 5.53 \\
& \text { 4b: Gef. C } 79.95 \text { H } 7.53 \text { N } 5.78 \\
& \text { 5b (+20\% 4b): Gef. C } 80.17 \text { H } 7.72 \text { N } 5.68
\end{aligned}
$$

Umsetzung von 1 mit 4-Methyl-4H-1,2,4-triazol-3,5-dion (MTAD): $4.98 \mathrm{~g}$ (54.1 mmol) 1 in $500 \mathrm{ml}$ Ether wurden mit $200 \mathrm{ml}$ absol. Dichlormethan verdünnt, im Eisbad gekuhlt, gerührt und unter Stickstoff portionsweise mit $6.16 \mathrm{~g}(54.5 \mathrm{mmol}) \mathrm{MTAD}^{28)}$ versetzt, wobei man den Kolben mit einer Aluminiumfolie vor Lichteinwirkung schützte und vor der neuen Zugabe jeweils das Ausbleichen der Rotfarbung abwartete (insgesamt $1 \mathrm{~h}$ ). Nach einer weiteren h bei $0^{\circ} \mathrm{C}$ engte man i. Vak. ein. Es blieben $11.84 \mathrm{~g}$ eines braunen Öls zuruck. Die Ausbeutebestimmung sofort nach der Darstellung anhand des $400-\mathrm{MHz}^{-1} \mathrm{H}-\mathrm{NMR}$-Spektrums mit Mesitylen als internem Standard ergab $2.35 \mathrm{~g}(21 \%)$ 1,N-Dimethyl-7,8-diazatetracyclo[3.3.0.0 2.4. $0^{3.6}$ Joctan-7,8-dicarboximid (7), $0.90 \mathrm{~g}(8 \%)$ 4,N-Dimethyl-7,8-diazatetracyclo[3.3.0.02.4.0.6.6 $/$ octan-7,8-dicarboximid (6) und $0.90 \mathrm{~g}(8 \%$ ) 2,N-Dimethyl-3,4-diazatricyclo[3.3.0.02,6 Joct-7-en-3,4-dicarbaximid (8). 2,N-Dimethyl-3,4-diazatricyclo[3.3.0.02,8 Joct-6-en-3,4-dicarboximid (9) war unter diesen Bedingungen nicht nachweisbar. Mehrere Tage bei $20^{\circ} \mathrm{C}$ aufbewahrte Proben zeigten die zunehmende Bildung von 9 auf Kosten von 8 an. Die Reinigung erfolgte durch Săulenchromatographie an neutralem Aluminiumoxid (Akt.-Stufe III) mit Ether als Laufmittel. Die erste Fraktion enthielt 8 und je nach Dauer der Operation wechselnde Mengen 9; in der zweiten Fraktion fanden sich 6 und 7 und je nach Alter der eingesetzten Probe wechselnde Mengen 9. Eine rasche Chromatographie der ersten Fraktion an Kieselgel mit Ether (Chromatotron) erbrachte die saubere Trennung von 8 (kurzere Retentionszeit) und 9 (längere Retentionszeit, farblose Kristalle, Schmp. $101-102^{\circ} \mathrm{C}$ ).

Reines 8, gelठst in $\mathrm{CDCl}_{3}$, war bei $20^{\circ} \mathrm{C}$ nach $6 \mathrm{~d}$ in ein Gemisch aus $32 \% 8$ und $68 \% 9$ und nach $13 \mathrm{~d}$ in ein solches aus $7 \% 8$ und $93 \% 9$ übergegangen. In $\left(\mathrm{CD}_{3}\right)_{2} \mathrm{SO}$ entstand aus reinem 8 bei $20^{\circ} \mathrm{C}$ in $6 \mathrm{~d}$ ein Gemisch aus $50 \% 8$ und 50\% 9. Auch kristallisiertes 8 lagerte sich in 9 um, allerdings nur mit $1 / 30$ der in $\mathrm{CDCl}_{3}$-Losung beobachteten Geschwindigkeit.

MS (70 eV): Gemisch aus 6, 7 und wenig 9: $m / z=205\left(11 \%, \mathrm{M}^{+}\right), 148(26), 147(26), 121$ (18), 107 (49), 105 (16), 94 (21), 93 (25), 92 (49), 91 (100), 80 (17), 79 (34), 65 (23), 52 (23), 51 (18), 39 (30). - Gemisch aus 8 und $9: m / z=205\left(14 \%, M^{+}\right), 148$ (18), 121 (11), 107 (100), 91 (31), $83(16), 79(34), 65(12), 52(15), 39(14)$. - Reines 9: $\mathrm{m} / z=205\left(24 \%, \mathrm{M}^{+}\right), 148(21), 107(100)$, 91 (28), 83 (12), 79 (33), 65 (12), 52 (12), 39 (12). 
${ }^{1} \mathrm{H}-\mathrm{NMR}\left(\mathrm{CDCl}_{3}\right): 6: \delta=1.47\left(\mathrm{~s} ; \mathrm{C}-\mathrm{CH}_{3}\right), 2.18$ (d von AA'-Teil, $J_{2,3}=4.8, J_{1,2}+J_{1,3}=$ $2.5 \mathrm{~Hz} ; 2-, 3-\mathrm{H}$ ), $\approx 3.03$ (überlagert durch $\mathrm{NCH}_{3}$-Signal; in $\mathrm{C}_{6} \mathrm{D}_{6}$ keine Uberlagerung, quint bei 2.22; 5-H), $3.02\left(\mathrm{~s} ; \mathrm{NCH}_{3}\right.$ ), 4.95 (d von XX'-Teil, $\left.J_{1,5}=4.5 \mathrm{~Hz} ; 1-, 6-\mathrm{H}\right) .-7: \delta=1.95(\mathrm{~s} ;$ $\left.\mathrm{C}-\mathrm{CH}_{3}\right), 2.26\left(\approx \mathrm{q}, J_{2,3}=J_{2,5}=4.4 \mathrm{~Hz} ; 2-\mathrm{H}\right), 2.37(\mathrm{~m} ; 3 \cdot \mathrm{H}), 2.75\left(\mathrm{td}, J_{2,4}=J_{3,4}=3.3\right.$, $J_{4,5}=0.9 \mathrm{~Hz} ; 4-\mathrm{H}$ ), $\approx 3.07$ (uberlagert durch $\mathrm{NCH}_{3}$-Signal; in $\mathrm{C}_{6} \mathrm{D}_{6}$ keine Uberlagerung, qd bei $\left.2.25, J_{3,5}=4.6, J_{5,6}=4.7 \mathrm{~Hz} ; 5-\mathrm{H}\right), 3.07\left(\mathrm{~s} ; \mathrm{NCH}_{3}\right), 4.94\left(\mathrm{dd}, J_{3,6}=2.2 \mathrm{~Hz} ; 6-\mathrm{H}\right) .-8$ : $\delta=1.67\left(\mathrm{~s} ; \mathrm{C}-\mathrm{CH}_{3}\right), 2.52\left(\mathrm{t}, J_{1,7}+J_{1,8}=4.5 \mathrm{~Hz} ; 1-, 6-\mathrm{H}\right), 3.08\left(\mathrm{~s} ; \mathrm{NCH}_{3}\right), 5.25(\mathrm{~s} ; 5-\mathrm{H}), 6.34$ $(\mathrm{t} ; 7-, 8-\mathrm{H})$, aus den ${ }^{13} \mathrm{C}$-Satelliten ergaben sich $J_{\mathrm{C}-7,7-\mathrm{H}}=176, J_{7,8}=5.8, J_{\mathrm{C}-1,1-\mathrm{H}}=156$, $J_{1,6}=8.8 \mathrm{~Hz} .-9: \delta=1.68\left(\mathrm{~s} ; \mathrm{C}-\mathrm{CH}_{3}\right), 2.19\left(\mathrm{dd}, J_{1,8}=6.3, J_{7,8}=2.5 \mathrm{~Hz} ; 8-\mathrm{H}\right), 2.98(\mathrm{~s}$; $\mathrm{NCH}_{3}$ ), $3.07(\approx t ; 1-\mathrm{H}), 5.17$ (dd, $\left.J_{1,5}=5.3, J_{5,6}=1.8 \mathrm{~Hz} ; 5-\mathrm{H}\right), 5.64$ (dd, $J_{6,7}=5.4 \mathrm{~Hz} ; 6-\mathrm{H}$ ), 6.14 (br. dd; 7-H).

${ }^{13} \mathrm{C}-\mathrm{NMR}\left(\mathrm{CDCl}_{3}\right): 6: \delta=10.89\left(\mathrm{q} ; 4-\mathrm{CH}_{3}\right), 23.45$ (d; C-2, -3$), 24.93(\mathrm{~s} ; \mathrm{C}-4), 25.35$ (q; $\mathrm{NCH}_{3}$ ), 51.05 (d; C-5), 60.62 (d; C-1, -6), 157.42 (s; C = O). - 7: $\delta=15.38$ (d; C-4), 15.93 (q; 1- $\mathrm{CH}_{3}$ ), 20.77 (d; C-3), 23.96 (d; C-2), 25.35 (q; $\mathrm{NCH}_{3}$ ), 55.63 (d; C-5), 60.48 (d; C-6), 71.19 (s; C-1), 155.82 und 155.92 (oder 157.42, jeweils s; $2 \mathrm{C}=0$ ). $-8: \delta=8.98$ (q; $2-\mathrm{CH}_{3}$ ), 25.72 (q; $\mathrm{NCH}_{3}$ ), 56.84 (d; C-1, -6), 91.05 (d; C-5), 105.98 (s; C-2), 131.21 (d; C-7, -8), 157.15 und 157.33 (jeweils s; $2 \mathrm{C}=0$ ). -9 (Zuordnung mit Hilfe des Spektrums von 9a,b, siehe unten): $\delta=18.78$ (q; 2- $\mathrm{CH}_{3}$ ), 25.28 (q; $\mathrm{NCH}_{3}$ ), 41.09 (d; C-8), 42.19 (d; C-1), 53.35 (s; C-2), 69.25 (d; C-5), 128.97 (d; C-6), 135.27 (d; C-7), 154.54 und 156.67 (jeweils s; $2 \mathrm{C}=0$ ).

$\mathrm{C}_{10} \mathrm{H}_{11} \mathrm{~N}_{3} \mathrm{O}_{2}$ (205.2) Ber. C 58.53 H $5.40 \mathrm{~N} 20.486+7$ : Gef. C 58.53 H $5.41 \mathrm{~N} 20.24$ 8 + 9: Gef. C 58.74 H 5.59 N 20.34 9: Gef. C 59.18 H 5.48 N 20.48

Umsetzung von 1a mit MTAD: Analog zu voranstehender Vorschrift setzte man $590 \mathrm{mg}$ (6.34 mmol) 1 a mit $716 \mathrm{mg}$ (6.34 mmol) MTAD um und erhielt nach Chromatographie an Kieselgel (Säule $30 \times 2.5 \mathrm{~cm}$ ) mit Ether $150 \mathrm{mg}(12 \%) 8 \mathrm{a}$ und $9 \mathrm{a}, \mathrm{b}$ im Verhaltnis $3: 1$ und $250 \mathrm{mg}$ (19\%) 6a, b, 7a und 9a,b im Verhältnis $1: 6: 1$. Nach der weitgehenden Umlagerung von 8a wurde durch Chromatographie reines $9 \mathrm{a}, \mathrm{b}$ isoliert, Schmp. $105^{\circ} \mathrm{C}$. - MS $(70 \mathrm{eV}): \mathrm{m} / z=206$ $\left(27 \%, \mathrm{M}^{+}\right), 205\left(0.4, \mathrm{M}^{+}\right.$von 9), $149(27), 108(100), 92(23), 80(30) .-{ }^{13} \mathrm{C}-\mathrm{NMR}\left(\mathrm{CDCl}_{3}\right)$, die Doppelsignale zeigen unterschiedliche Deuteriumisotopeneffekte in den Isotopomeren an: $\delta=18.71$ und 18.75 (jeweils $q ; 2-\mathrm{CH}_{3}$ ), $25.28\left(q ; \mathrm{NCH}_{3}\right.$ ), 40.94 (d; C-8), 42.03 und 42.14 (jeweils d; C-1), 53.25 und 53.33 (jeweils s; C-2), 69.21 (d; C-5), 128.78 und 128.93 (jeweils d; C-6), 135.18 (d; C-7), 154.54 und 156.67 (jeweils s; $2 \mathrm{C}=0$ ).

${ }^{1} \mathrm{H}$-NMR $\left(\mathrm{CDCl}_{3}\right)$ : Im Vergleich zu den Spektren der nicht markierten Verbindungen ergaben sich folgende Veränderungen: $6 \mathrm{a}, \mathrm{b}$ : Die Intensitat der Signale bei $\delta=2.18$ (br. d, $J_{2,5}=4.8 \mathrm{~Hz}$; $2-, 3-\mathrm{H}$ ) und 4.95 (dd, $J_{5,6}=4.5$, weiterer Linienabstand $\approx 2 \mathrm{~Hz}$, uberlagert von d, $J_{1,5}=4.5 \mathrm{~Hz}$; 1-, 6-H) war auf jeweils $\approx 75 \%$ abgesunken; Multiplizitatsänderung beim 5-H-Signal wegen Überlagerung durch $\mathrm{NCH}_{3}$-Bande nicht erkennbar. - 7a: Das Signal bei $\delta=2.75$ war fast vollständig verschwunden; $\delta=2.26(\mathrm{t} ; 2-\mathrm{H}), 2.37(\mathrm{td} ; 3-\mathrm{H}) . \quad-8 \mathrm{a}$ : Die Intensitat des Signals bei $\delta=6.34$ war auf $\approx 50 \%$ reduziert; $\delta=2.52$ (d, Linienabstand $2.3 \mathrm{~Hz} ; 1-, 6-\mathrm{H})$. Intensităt der Signale bei $\delta=2.19\left(\mathrm{~d}, J_{1,8}=6.3 \mathrm{~Hz} ; 8-\mathrm{H}\right)$ und $6.14\left(\mathrm{~d}, J_{6,7}=5.4 \mathrm{~Hz} ; 7-\mathrm{H}\right)$ war auf $\approx 50 \%$ reduziert; $\delta=5.64$ (dd, $J_{6,7}=5.3, J_{5,6}=1.8 \mathrm{~Hz}$, uberlagert von d, $J_{5,6}=1.8 \mathrm{~Hz}$; $6-\mathrm{H}), 3.07\left(\approx \mathrm{t}, J_{1,8}=6.3, J_{1,5}=5.3 \mathrm{~Hz}\right.$, uberlagert von $\left.\mathrm{d}, J_{1,5}=5.3 \mathrm{~Hz} ; 1-\mathrm{H}\right)$.

I-(I-Methyl-(E)-2-pentenyl)- (17) und I-(I-Ethenylbutyl)-1,2-dihydro-4-methyl-4H-1,2,4triazol-3,5-dion (18): Gab man zur Losung von 1 uberschüssiges MTAD, so zeigten die Spektren des Rohprodukts die Gegenwart von 17 und 18 im Verhăltnis 3:2 an. Bei der Chromatographie wurden 17 und 18 mit Ether nicht eluiert. In einem Versuch setzte man 1 und MTAD aquimolar ein und destillierte dann i. Vak. alle flüchtigen Komponenten ab, unter denen 2-Hexen anhand der ${ }^{1}$ H-NMR-Signale seiner olefinischen Protonen nachgewiesen wurde. Die mit dem Destillat ausge-

Chem. Ber. 119 (1986) 
führte Reaktion mit MTAD erbrachte nun 17 und 18 frej von 1-Addukten. - 'H-NMR ( $\left.\mathrm{CDCl}_{3}\right)$ : 17: $\delta=1.00\left(\mathrm{t}, J_{4,5}=7.5 \mathrm{~Hz} ; 5-\mathrm{H}_{3}\right), 1.36\left(\mathrm{~d}, J_{1^{\prime}, 1}=6.5 \mathrm{~Hz} ; 1^{\prime}-\mathrm{H}_{3}\right), 2.06$ (br. quint; 4-H $), 3.06$ (s; $\mathrm{NCH}_{3}$ ), 4.74 (br. quint; 1-H), 5.50 (ddt, $J_{2,3}=15.5, J_{1,2}=6.5, J_{2,4}=1.6 \mathrm{~Hz} ; 2-\mathrm{H}$ ), 5.78 (dtd, $\left.J_{3,4}=6.4, J_{1,3}=1.3 \mathrm{~Hz} ; 3-\mathrm{H}\right), 9.7$ (sehr breit; NH). $18: \delta=0.96\left(\mathrm{t}, J_{3,4}=7.4 \mathrm{~Hz} ; 4-\mathrm{H}_{3}\right), 1.36$ $\left(\mathrm{m} ; 3-\mathrm{H}_{2}\right), 1.66$ und 1.78 (jeweils $\left.\mathrm{m} ; 2-\mathrm{H}_{2}\right), 3.07\left(\mathrm{~s} ; \mathrm{NCH}_{3}\right), 4.60\left(\mathrm{br} . \mathrm{q}, J_{1,2}=7.7 \mathrm{~Hz} ; 1-\mathrm{H}\right), 5.23$

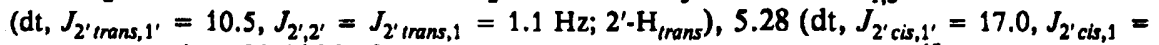
$1.1 \mathrm{~Hz} ; 2^{\prime}-\mathrm{H}_{c i s}$ ), 5.83 (ddd, $\left.J_{1^{\prime}, 1}=7.0 \mathrm{~Hz} ; 1^{\prime}-\mathrm{H}\right), 9.7$ (sehr breit; NH). - ${ }^{13} \mathrm{C}-\mathrm{NMR}\left(\mathrm{CDCl}_{3}\right)$ : $17: \delta=13.2(q ; C-5), 17.4(q ; C-1), 25.1\left(q ; N_{3} C_{3}\right), 25.3(t ; C-4), 54.0(d ; C-1), 126.3$ (d; C-3), 136.2 (d; C-2), 154.0 und 155.5 (jeweils s; $2 C=0$ ). 18: $\delta=13.6$ (q;C-4), $19.2(t ; C-3), 25.1$ (q; $\mathrm{NCH}_{3}$ ), 33.7 (t; C-2), 58.7 (d; C-1), 118.4 (t; C-2), 134.8 (d; C-1'), 154.2 und 155.5 (jeweils s; $2 \mathrm{C}=0$ ).

1) Aus der Dissertation B. Mattauch, Univ. Wurzburg 1985.

2) T. J. Katz, E. J. Wang und N. Acton, J. Am. Chem. Soc. 93, 3782 (1971); T. J. Katz, R. J. Roth, N. Acton und E. J. Carnahan, Org. Synth. 53, 157 (1973).

3) Ubersicht: $M$. Christl, Angew. Chem. 93, 515 (1981); Angew. Chem., Int. Ed. Engl. 20, 529 (1981).

4) 4a) $M$. Christl und $M$. Lechner, Chem. Ber. 115, 1 (1982). - 4b) $H$. Leininger, $F$. Lanzendorfer und $M$. Christl, Chem. Ber. 116, 669 (1983). - 4c) H. Leininger, M. Christl und D. Wendisch, Chem. Ber. 116, 681 (1983). - 4d) M. Christl, E. Brunn und F. Lanzendorffer, J. Am. Chem. Soc. 106, 373 (1984).

5) A.-D. Schluter, J. Belzner, U. Heywang und G. Szeimies, Tetrahedron Lett. 24, 891 (1983); Dissertation $A .-D$. Schluter, Univ. München 1984.

6) 6a) H. Leininger, P. Kemmer, K. Beck und M. Christl, Chem. Ber. 115, 3213 (1982). -

6b) $M$. Christl und $R$. Lang, J. Am. Chem. Soc. 104, 4494 (1982). - 6c) $F$. Lanzendorfer und M. Christl, Angew. Chem. 95, 896 (1983); Angew. Chem., Int. Ed. Engl. 22, 871 (1983). 6d) M. Christl, H. Leininger und P. Kemmer, Chem. Ber. 117, 2963 (1984).

7) T. J. Katz und K. C. Nicolaou, J. Am. Chem. Soc. 96, 1948 (1974).

8) $U$. Burger und G. Gandillon, Tetrahedron Lett. 1979, 4281; U. Burger, G. Gandillon und J. Mareda, Helv. Chim. Acta 64, 844 (1981).

9) M. Christl, B. Mattauch, H. Irngartinger und A. Goldmann, Chem. Ber. 119, 950 (1986), vorstehend.

10) D. Seyferth und F. M. Armbrecht jr., J. Organomet. Chem. 16, 249 (1969).

11) $R$. Huisgen und $U$. Burger, Tetrahedron Lett. 1970, 3053.

12) $M$. Christl und G. Brintrup. Chem. Ber. 107, 3908 (1974).

13) $M$. Christl und $R$. Herbert, Org. Magn. Reson. 12, 150 (1979).

14) $M$. Christl und S. Freund, Chem. Ber. 118, 979 (1985).

15) M. Christl, Chem. Ber. 108, 2781 (1975).

16 K. Bast, M. Christl, R. Huisgen und W. Mack, Chem. Ber. 106, 3312 (1973).

in T. J. Katz und N. Acton, J. Am. Chem. Soc. 95, 2738 (1973).

18) $M$. Christl und B. Mattauch, Chem. Ber. 118, 4203 (1985).

19) M. Christl, H. Leininger und B. Mattauch, Spectrosc. Int. J. 2, 184 (1983).

20) $H$. M. Frey und $R$. G. Hopkins, J. Chem. Soc. B $1970,1410$.

21) J. Meinwald und D. Schmidt, J. Am. Chem. Soc. 91, 5877 (1969); J. Meinwald und H. Tsuruta, ebenda 91, 5877 (1969); H. E. Zimmerman, J. D. Robbins und J. Schantl, ebenda 91, 5878 (1969).

22) M. J. Goldstein und R. Hoffmann, J. Am. Chem. Soc. 93, 6193 (1971).

23) $D$. M. Lemal und J. P. Lokensgard, J. Am. Chem. Soc. 88, 5934 (1966).

24) S. Ohashi, K. Leong, K. Matyjaszewski und G. B. Butler, J. Org. Chem. 45, 3467 (1980); S. Ohashi und G. B. Butler, ebenda 45, 3472 (1980).

25) CHEMETALL, Frankfurt/Main.

26) A. Werner und H. Buss, Ber. Dtsch. Chem. Ges. 27, 2193 (1894); K.-C. Liu, B. R. Shelton und $R$. K. Howe, J. Org. Chem. 45, 3916 (1980).

27) C. Grundmann und J. M. Dean, J. Org. Chem. 30, 2809 (1965).

28) J. C. Stickler und W. H. Pirkle, J. Org. Chem. 31, 3444 (1966); R. C. Cookson, S. S. Gupte, I. D. R. Stevens und C. T. Watts, Org. Synth. 51, 121 (1971).

[175/85]

Chem. Ber. 119 (1986) 